\title{
Culicideofauna (Diptera) encontrada em entrenós de taquara de uma mata residual na área urbana de Londrina, Paraná, Brasil
}

\author{
João Antonio Cyrino Zequi ${ }^{1}$ \\ José Lopes ${ }^{2}$
}

\begin{abstract}
Fauna of Culicidae (Diptera) found in the internodes of the bamboo in a residual forest in the urban area of Londrina - Paraná, Brazil. A fragment of a forest, located in the urban area of Londrina, Paraná, Brazil,was chosen to represent the area for this study. Many Merostachys Spreng, 1824 bamboo, which were naturally perforated and, therefore, ready to retain water, were found in the interior of this forest. Bamboo were collected every fifteen days during field actions. They were then measured and cut meter by meter before their contents were analyzed in the laboratory. A total of 542 specimens from the following species were collected during one year: Sabethes aurescens (Lutz, 1905), Sabethes melanomymphe Dyar, 1924, Sabethes identicus Dyar \& Knab, 1907, Wyeomyia rooti (Del Ponte, 1939), Wyeomyia limai Lane \& Cerqueira, 1942, Wyeomyia sp., Trichoprosopon pallidiventer (Lutz, 1905), Trichoprosopon humboldti Lane \& Cerqueira, 1942, Toxorhynchites sp. e Shannoniana schedocyclium (Dyar \& Knab, 1908). Wyeomyia limai and Sabethes aurescens were the most frequently found species. The first specie had the highest population index in february whereas the Sabethes aurescens, had its highest index in july, february and november. The largest number of larvae were obtained in the months of february and march. As for stratification, the greatest quantity of larvae was found at two meters. The specie Wyeomyia limai predominated in all heights, being most abundant at three meters high whereas the Sabethes was most abundant at one meter high. The presence of bamboo in small forest spots in urban areas is enough to host a certain variety of Culicidae of the Sabethini tribe which are considered wild mosquito. These same mosquito, due to their hematophagus habitat, can be hazardous to public health.
\end{abstract}

KEY WORDS. Culicidae, larvae, bamboo internodes, natural breeding place

De acordo com JENKINS \& CARPENTER (1946), os buracos nos troncos de árvores são, provavelmente, o mais antigo tipo de criadouro estabelecido para Culicidae. Colmos vivos de taquaras ou bambus são um dos mais especializados habitats para larvas de mosquito (MACDONALD \& TRAUB 1960).

Taquara e bambu são nomes populares de alguns gêneros de Gramineae, que são nativos de regiões tropicais e subtropicais (Lozover 1998). Para a oviposição na água retida nos entrenós destas plantas, espécies de culicídeos desenvolveram estratégias altamente especializadas, como em Sabethes chloropterus (Von Hum-

1) Curso de Pós-graduação em Agronomia, Centro de Ciências Agrárias, Universidade Estadual de Londrina. Caixa Postal 6001, 86051-970 Londrina, Paraná, Brasil. E-mail: joao.zequi@usa.net

2) Departamento de Biologia Animal e Vegetal, Universidade Estadual de Londrina. Caixa Postal 6001, 86051-970 Londrina, Paraná, Brasil. E-mail: jea@uel.br 
boldt, 1819), o vetor do vírus da febre amarela na América Central, que deposita seus ovos pelos pequenos orifícios no bambu. $\mathrm{O}$ mosquito reconhece o criadouro visualmente, e dispara os ovos nos orifícios até uma distância aproximada de $10 \mathrm{~cm}$ (MACHADO-Allison 1980).

O acesso de Culicidae ao interior dos entrenós é facilitado pela ação de insetos que perfuram esse tipo de colmo (DAVIS 1944; COSTA LIMA 1956; BORROR \& DeLonG 1969; LouTON et al. 1996; Lozovei 1998). O acúmulo de água nos entrenós é decorrente de água de chuva que escorre pelo caule do bambu e penetra pelos orifícios feitos pelos insetos; esta água conduz ainda partículas orgânicas que servirão diretamente de alimento para as larvas ou propiciarão o desenvolvimento de microrganismos, base da alimentação de muitas larvas filtradoras. LOUTON et al. (1996) afirmaram que outra forma de acúmulo de líquido nos entrenós é feita pela retenção da água metabólica, de composição química diferente da água continental, produzida pelos colmos que atingem o estágio vegetativo pleno.

Espécies de mosquitos que proliferam nestes habitats são consideradas silvestres e, entre elas, aparecem algumas que são importantes por serem transmissoras de agentes patogênicos para o homem e outros animais. Esta importância aumenta quando esses criadouros estão localizados em área urbana ou periurbana. Os culicídeos da tribo Sabethini e subfamília Toxorhynchitinae são os principais colonizadores deste tipo de habitat.

Existe escassa informação sobre a oviposição dos sabetíneos e, aparentemente, a maioria deles deposita os ovos de forma individual, sobre a superfície da água. Agentes virais têm sido isolados em mosquitos deste grupo coletados na natureza, os quais são vetores de vírus da febre amarela e das encefalites.

Toxorhynchitinae inclui os mosquitos de porte avantajado, multicoloridos e não hematófagos. Desenvolvem-se em bromélias, buracos de árvores e entrenós de bambu ou em criadouros artificiais como latas, barris, pneumáticos e outros (LOPES 1997).

No presente trabalho objetivou-se levantar e identificar as espécies de mosquitos que procriam em entrenós de taquara verde, no interior de um fragmento de mata localizado na área urbana de Londrina, Paraná, bem como verificar a influência da temperatura ambiente e precipitação pluviométrica sobre esta entomofauna.

\section{MATERIAL E MÉTODOS}

As coletas em campo foram realizadas no período de setembro 1995 a setembro de 1996, em uma reserva florestal com cerca de 3,3 hectares, conhecida como Mata Daher, $573 \mathrm{~m}$ de altitude, localizada na área urbana de Londrina, Paraná, Brasil, próximo a rodovia Celso Garcia Cid, PR 445, Km 300 (2318'55"S; $51^{\circ} 12^{\prime} 16^{\prime \prime W}$ ) (Fig. 1). É uma mata alterada, onde ainda existem árvores nativas de grande porte. No estrato inferior, encontra-se grande quantidade de cipós e abundância de taquara do gênero Merostachys Spreng, 1824 como vegetação secundária, e em uma das margens da mata corre o Ribeirão Cambézinho (Fig. 1). As taquaras encontravam-se, com maior freqüência, próximo à margem de uma nascente de água formando grandes concentrações. 


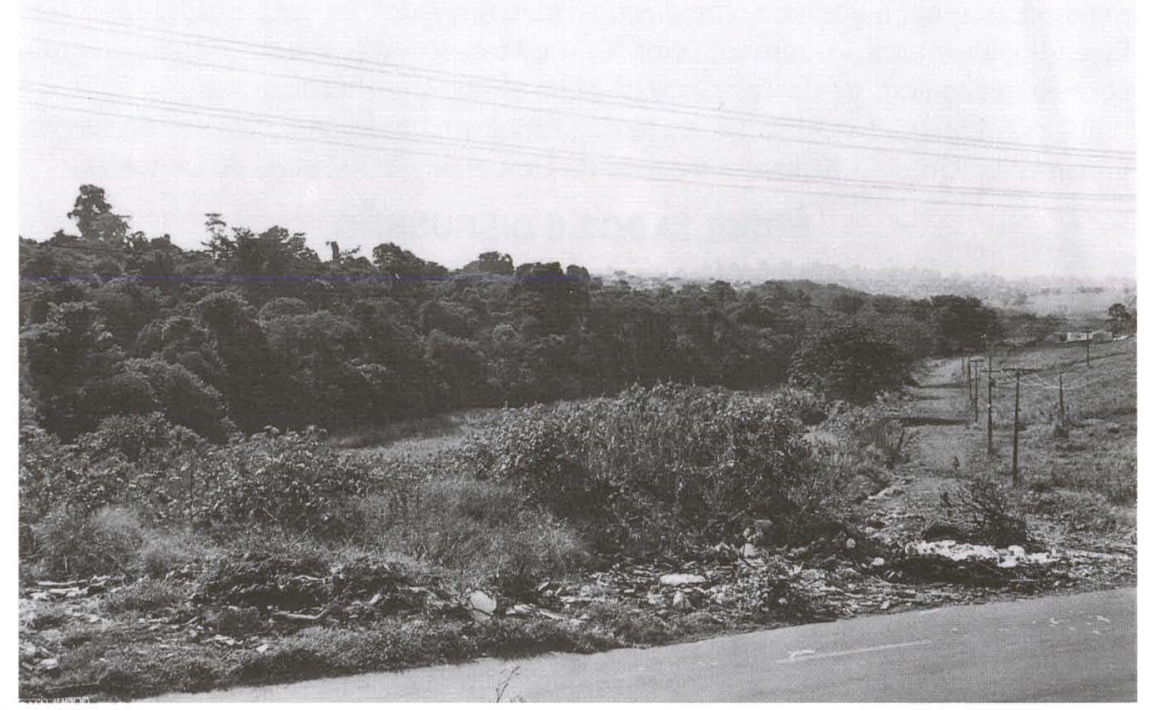

Fig. 1. Área de coleta de culicídeos, localizada na área urbana de Londrina, Paraná (Mata Daher).

Cinco colmos perfurados de taquara verde, foram coletados quinzenalmente; ao acaso. Adentrava-se à mata e caminhando entre as taquaras, retirando-se exemplares maduros e que continham furos, sem outros critérios de escolha. Antes da taquara ser cortada rente ao solo, os orifícios eram localizados e tapados com fita adesiva, para não extravasar o material biológico de interesse.

Os colmos foram medidos e seccionados transversalmente a cada metro, até a altura de cinco metros. Cada metro do colmo foi aberto longitudinalmente, sobre uma bandeja, separando-se duas metades, e a água presente no interior dos entrenós escorria para a bandeja. Com auxílio de pissete, as metades eram lavadas dentro desta bandeja onde as larvas foram coletadas. O líquido, contendo aproximadamente $300 \mathrm{ml}$ foi guardado em potes plásticos, sendo um para cada altura para posterior revisão, já que larvas de primeiro estágio ou ovos poderiam passar desapercebidos.

Os culicídeos imaturos foram separados em copos plásticos de $50 \mathrm{ml}$, conforme a altura da taquara, utilizando-se a mesma água do criadouro. Os recipientes contendo as larvas foram mantidas no escuro. Quando atingiam a fase de pupa, coletava-se as exúvias de quarto ínstar, as quais foram montadas em lâmina permanente, utilizando-se Bálsamo do Canadá, para posterior auxílio na identificação dos seus respectivos adultos. As identificações foram feitas utilizando-se as chaves contidas em LANE (1953), ForATTINI (1965) e KNIGHT \& STONE (1977).

Os dados de temperatura e precipitação pluviométrica foram obtidos através da estação meteorológica do Instituto Agronômico do Paraná (IAPAR), distante aproximadamente $3 \mathrm{~km}$ da área do experimento. 
Os resultados foram submetidos à análise de variância com os valores relacionados ao número de indivíduos transformado em raiz quadrada $+0,5$, fazendo-se a interação entre espécies X meses e espécies X altura. As médias foram comparadas pelo teste de Tukey no nível de 5\% de significância.

Os culicídeos coletados estão depositados na coleção de insetos do Departamento de Biologia Animal e Vegetal da Universidade Estadual de Londrina.

\section{RESULTADOS E DISCUSSÃO}

Foram coletadas 542 larvas de Culicidae, pertencentes a 5 gêneros: Wyeomyia Theobald, 1901 (55,4\%), Sabethes Robineau-Desvoidy, 1827 (38,6\%), seguidos de Trichoprosopon Theobald, 1901 (3,0\%), Toxorhynchites Theobald, 1901 $(2,2 \%)$ e Shannoniana Lane \& Cerqueira, 1903 (0,9\%) (Tab. I).

A tabela I relaciona as espécies quantificando-as de acordo com a altura do colmo em que foram coletadas. Wyeomyia (Wyeomyia) limai Lane \& Cerqueira, 1942 foi a mais freqüente e abundante, contribuindo com $49,6 \%$ do total. Sabethes (Peytonulus) aurescens (Lutz, 1905) foi a segunda espécie mais abundante, com $29,7 \%$ dos imaturos coletados. LozOver (1998), em estudos com taquaras verdes na Serra do Mar e Primeiro Planalto do Paraná, também encontrou essas duas espécies como mais abundantes, mas em ordem invertida do observado aqui. Estes dois gêneros são freqüentemente coletados em estudos que envolvam taquaras e buracos de árvores (DA VIS 1944; LOZOVEI \& LUZ 1976; LOUTON et al. 1996). DAVIS (1944) coletando em bambu no estado do Rio de Janeiro, obteve mais exemplares de Sabethes identicus Dyar \& Knab, 1907 (Sabethes (Sabethinus) lutzanus, Lane \& Cerqueira, 1942) do que Sabethes aurescens.

No estrato vertical de um a dois metros ocorreu a maior diversidade de espécies. Todas as espécies constatadas fizeram-se presentes neste estrato, e os imaturos coletados nesta altura correspondem a 29,7\% do total coletado. Trichoprosopon (Runchomyia) humboldti Lane \& Cerqueira, 1942 e Wyeomyia sp. restringiram as suas ocorrências neste estrato com 1,1\% (Tab. I). Estas espécies podem ser especialistas, não adaptadas para este hábitat ou para estas condições ambientais, já que foram pouco freqüentes.

Tabela I. Espécies de culicideos coletadas em diferentes alturas de taquara Merostachys no interior de mata situada na área urbana de Londrina, Paraná.

\begin{tabular}{|c|c|c|c|c|c|c|}
\hline \multirow{2}{*}{ Culicidae } & \multicolumn{6}{|c|}{ Alturas (m) } \\
\hline & $0-1$ & $1-2$ & $2-3$ & $3-4$ & $4-5$ & Total \\
\hline Sabethes aurescens & 50 & 48 & 28 & 21 & 14 & 161 \\
\hline Sabethes melanonymphe & 14 & 4 & 10 & 6 & 4 & 38 \\
\hline Sabethes identicus & 2 & 1 & 4 & 2 & 1 & 10 \\
\hline Wyeomyia rooti & 7 & 11 & 10 & 0 & 0 & 28 \\
\hline Wyeomyia limai & 46 & 76 & 85 & 43 & 19 & 269 \\
\hline Wyeomyia sp. & 0 & 3 & 0 & 0 & 0 & 3 \\
\hline Trichoprosopon pallidiventer & 2 & 6 & 2 & 2 & 1 & 13 \\
\hline Trichoprosopon humboldti & 0 & 3 & 0 & 0 & 0 & 3 \\
\hline Toxorhynchites sp. & 0 & 6 & 3 & 3 & 0 & 12 \\
\hline Shannoniana schedocyclium & 1 & 3 & 1 & 0 & 0 & 5 \\
\hline Total & 122 & 161 & 143 & 77 & 39 & 542 \\
\hline
\end{tabular}


A estratificação vertical das quatro espécies mais freqüentes consta na figura 2. Wyeomyia limai aumenta o número de indivíduos até os três metros de altura, ponto onde começa a diminuir (Fig. 2). O número de Sabethes aurescens diminui uniformemente com o aumento da altura, evidenciando sua ocorrência nos entrenós mais próximos do solo. Estas espécies, por serem as mais abundantes, podem ter determinado o padrão observado para os gêneros. Sabethes melanonymphe Dyar, 1924, predominou de 0-1 metro e sua população diminui a partir dos 3 metros de altura. Wyeomyia rooti (Del Ponte, 1939) foi mais abundante de 1-3 metros, não aparecendo nos estratos superiores.

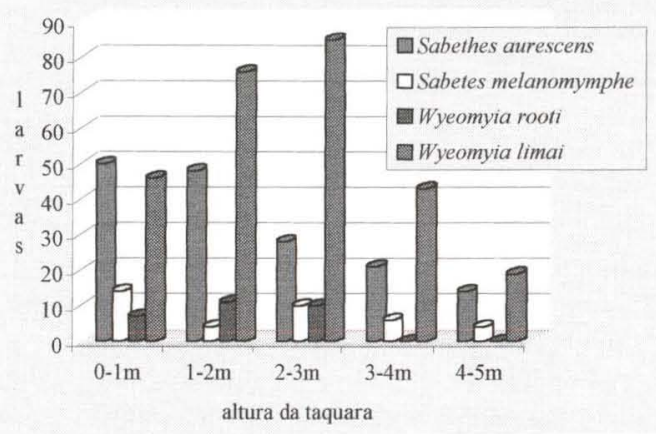

Fig. 2. Distribuição das quatro espécies de mosquitos mais freqüentes, conforme a altura dos entrenós de taquara, coletados em mata da área urbana de Londrina, Paraná, 1995/96.

Os resultados podem indicar diferenças na forma de exploração do habitat, evitando a competição por alimento e até mesmo pelo criadouro, já que esse hábitat, pode ser considerado altamente especializado onde os fatores limitantes são muito evidentes. Esta estratificação diferenciada pode também evitar a coexistência das espécies, prevenindo a predação ocasional entre elas. Os outros gêneros e espécies foram menos abundantes, mas para Trichoprosopon existe alguma evidência de colonização dos entrenós no estrato de 1-2 metros.

Para comprovar essa distribuição vertical, aplicou-se a Análise de Variância. Os resultados não mostraram diferenças significativas entre as espécies e alturas (Tab. II). O teste de Tukey, no nível de $5 \%$ de significância (Tab. III), indica que as alturas de 1 a 3 metros não diferem entre si, para preferência de procriação dos culicídeos. As alturas menos colonizadas foram as de cinco e as de quatro metros. Porém só há diferença significativa entre as alturas 1,2 e 3 metros com a de cinco metros.

Analisando-se as populações de Sabethes aurescens e Wyeomyia limai, ao longo dos doze meses, e aplicando-se o teste de Tukey, no nível de 5\% de significância (Tab. IV), verifica-se que Sabethes aurescens tem a maior média populacional no mês de julho, inverno, quando a média mensal da temperatura foi de $16^{\circ} \mathrm{C}$ e precipitação pluviométrica de $8,1 \mathrm{~mm}$. Estes são os valores mais baixos 
de temperatura e precipitação ao longo de todo o período de coletas (Figs 3, 4). Todavia, a média de julho (inverno) não difere estatisticamente de novembro e fevereiro (verão) (Tab. IV). Lozovei \& Luz (1976) em coletas no Planalto de Curitiba em Bambusa Schreb, 1789 e Merostachys, encontraram Trichoprosopon pallidiventer (Lutz, 1905) e Sabethes aurescens na primavera, verão e outono, o que não vai em parte de encontro com os dados aqui apresentados. Sabethes aurescens foi abundante em entrenós vivos de taquara, sendo a espécie mais freqüente coletada por LOZOVEI (1998).

Tabela II. Análise de variância de culicídeos procriando em entrenós de taquara Merostachys $\mathrm{sp}$. em cinco diferentes alturas durante um ano de coleta. (GL) Graus de liberdade, (SQ) Soma de quadrados, $(Q M)$ quadrado médio, $(F)$ Teste $F,(P)$ probabilidade.

\begin{tabular}{lrrrrr}
\hline \multicolumn{1}{c}{ Causas da variaçắo } & GL & SQ & QM & F & $P$ \\
\hline Meses & 11 & 7,167 & 0,652 & 3,54 & 0,01 \\
Espécies & 3 & 15,331 & 5,110 & 27,75 & 0,01 \\
Altura & 4 & 3,869 & 0,967 & 5,25 & 0,01 \\
Espécies x altura & 12 & 2,546 & 0,212 & 1,15 & 0,05 \\
Meses x espécies & 33 & 12,554 & 0,380 & 2,07 & 0,01 \\
Residuo & 176 & 32,384 & 0,184 & & \\
\hline Total & 239 & 73,851 & & & \\
\hline
\end{tabular}

$\mathrm{CV} \%=39,89$.

Tabela III. Médias de quatro espécies de larvas mais freqüentes que se procriam em cinco diferentes alturas de taquara, e as associações resultantes do teste de Tukey.

\begin{tabular}{cc}
\hline Altura $(\mathrm{m})$ & Média larval \\
\hline 1 & $1,209 \mathrm{a}$ \\
2 & $1,157 \mathrm{a}$ \\
3 & $1,144 \mathrm{a}$ \\
4 & $1,012 \mathrm{ab}$ \\
5 & $0,858 \mathrm{~b}$ \\
\hline
\end{tabular}

$\mathrm{CV} \%=39,89 ; \mathrm{DMS}=0,24 ;\left(^{*}\right)$ Médias seguidas pela mesma letra na coluna, não diferem entre si pelo teste de Tukey no nivel de $5 \%$ de significância.

Tabela IV. Médias mensal de quatro espécies de larvas mais freqüentes que se procriam em cinco diferentes alturas de taquara, e as associações resultantes do teste de Tukey.

\begin{tabular}{lcccc}
\hline Mês & Sabethes aurescens & Sabethes melanomymphe & Wyeomyia limai & Wyeomyia rooti \\
\hline Janeiro & 1,14 cbcd & $0,77 \mathrm{ab}$ & $1,66 \mathrm{bc}$ & $0,71 \mathrm{a}$ \\
Fevereiro & $1,57 \mathrm{ab}$ & $0,87 \mathrm{ab}$ & $2,43 \mathrm{a}$ & $1,10 \mathrm{a}$ \\
Março & $1,34 \mathrm{bcd}$ & $0,82 \mathrm{ab}$ & $1,88 \mathrm{~b}$ & $0,85 \mathrm{a}$ \\
Abril & $1,11 \mathrm{~cd}$ & $0,81 \mathrm{ab}$ & $1,44 \mathrm{bcde}$ & $0,82 \mathrm{a}$ \\
Maio & $0,93 \mathrm{~d}$ & $0,96 \mathrm{ab}$ & $1,81 \mathrm{bc}$ & $0,76 \mathrm{a}$ \\
Junho & $1,03 \mathrm{~cd}$ & $0,71 \mathrm{~b}$ & $1,72 \mathrm{bc}$ & $0,91 \mathrm{a}$ \\
Julho & $1,79 \mathrm{a}$ & $1,19 \mathrm{a}$ & $0,94 \mathrm{f}$ & $0,85 \mathrm{a}$ \\
Agosto & $1,10 \mathrm{~cd}$ & $0,83 \mathrm{ab}$ & $0,86 \mathrm{f}$ & $0,71 \mathrm{a}$ \\
Setembro & $0,97 \mathrm{~cd}$ & $0,75 \mathrm{ab}$ & $0,79 \mathrm{f}$ & $0,81 \mathrm{a}$ \\
Outubro & $1,02 \mathrm{~cd}$ & $0,88 \mathrm{ab}$ & $0,91 \mathrm{f}$ & $0,71 \mathrm{a}$ \\
Novembro & $1,41 \mathrm{abc}$ & $0,81 \mathrm{ab}$ & $1,05 \mathrm{def}$ & $0,71 \mathrm{a}$ \\
Dezembro & $1,28 \mathrm{bcd}$ & $0,88 \mathrm{ab}$ & $1,45 \mathrm{bcd}$ & $0,77 \mathrm{a}$ \\
\hline
\end{tabular}

$\mathrm{CV} \%=39,89 ; \mathrm{DMS}=0,44 ;\left(^{*}\right)$ Médias seguidas da mesma letra na coluna não diferem entre si pelo teste de Tukey no nível de $5 \%$ de significância. 


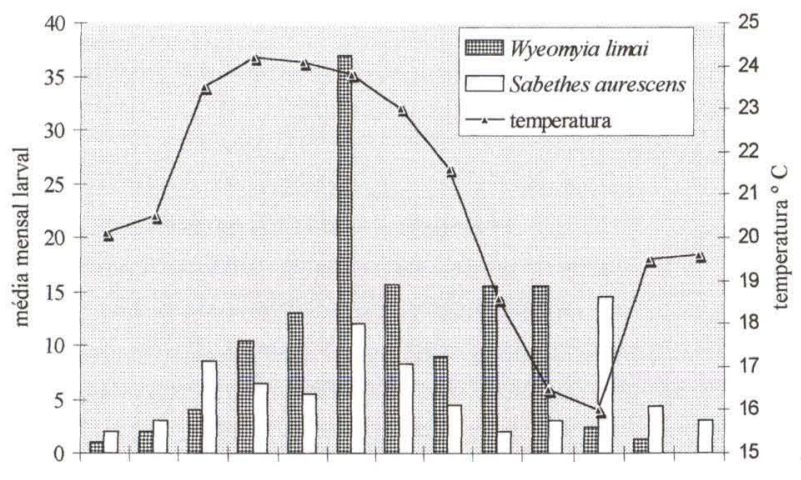

set out nov dez jan fev mar abr mai jun jul ago set

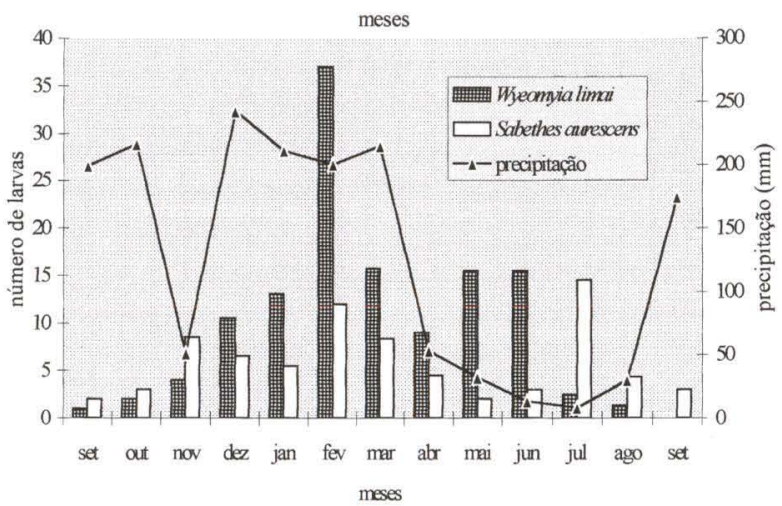

Figs 3-4. Média mensal de larvas de Sabethes aurescens e Wyeomyia limai, corelacionadas com a: (3) temperatura média mensal e (4) precipitação mensal, coletadas em mata da área urbana de Londrina, Paraná, 1995/96

Wyeomyia limai apresentou seu pico populacional em fevereiro, verão, com alta precipitação total $(200,1 \mathrm{~mm})$ e uma das maiores temperaturas médias registradas durante todo o ciclo de coleta $\left(23,8^{\circ} \mathrm{C}\right)$, como pode ser observado nas figuras 3 e 4. De acordo com a tabela IV, fevereiro foi a época mais propicia para reprodução desta espécie, e os meses menos favoráveis foram de julho a outubro. Esta espécie foi a segunda mais freqüente, em entrenós verdes, nas coletas realizadas por Lozover (1998), na Serra do Mar e no Primeiro Planalto do Paraná. Na presente pesquisa, esta espécie foi a mais freqüente, com $49,63 \%$ de imaturos coletados.

Wyeomyia rooti não mostrou variação populacional significativa durante 0 ano, mantendo-se em níveis estáveis ao longo de toda a pesquisa.

Sabethes melanomymphe apresentou-se praticamente constante ao longo do ano, todavia foi uma espécie pouco freqüente.

De acordo com os dados e análises realizadas, pode-se inferir que Wyeomyia limai e Sabethes aurescens apresentaram condições de habitats distintos, mas seria necessário uma análise mais minuciosa para a inferir sobre os fatores que interferem na flutuação dessas populações. 
DAvis (1944) verificou que Wyeomyia lutzi (Costa Lima, 1930), Wyeomyia oblita (Lutz, 1904), Sabethes aurescens e Sabethes lutzii Theobald, 1903 ovipuseram em taquaras com orifícios feitos por besouros; e que Trichoprosopon compressum Lutz, $1905 \mathrm{em}$ colmos de bambu perfurados a canivete. O mesmo autor verificou que Culex ocellatus Theobald, 1903 foi mais comum perto do chão enquanto que Trichoprosopon reversum Lane \& Cerqueira, 1942 e Phonomyia pilicauda (Root, 1928) são colonizadores de entrenós a maiores alturas.

Outro fator importante na dinâmica de população de insetos de pequenos reservatórios é a predação. SOTA \& MOGI (1996) verificaram que, na presença de larva predadora Toxorhynchites, a densidade e a biomassa de culicídeos, por amostra, reduzia significativamente em bambus analisados, no norte de Sulawesi (Indonésia). Estes autores estudaram em uma região com características tropicais (planície Kosinggolan) e em outra com características temperada (região montanhosa Moat) em Sulawesi, encontraram baixa taxa deste predador, sendo esta larva a única predadora. No presente estudo, Toxorhynchites ocorreu com baixo índice $(2,2 \%)$, sendo o único predador encontrado. LOPES (1999), estudando a influência de larvas predadoras em pneus instalados em mata ciliar no Norte do Paraná, verificou que a densidade populacional de Culex quinquefasciatus Say, 1823 e Culex eduardoi Casal \& Garcia, 1968 diminui significativamente, o que comprova a influência desse predador na dinâmica populacional de pequenos criadouros artificiais. A comunidade viva deste tipo de ecossistema é influenciada predominantemente pelas transformações biológicas da matéria orgânica morta, pela biomassa microbiana e também por insetos e por distúrbios esporádicos, representados principalmente por chuvas (BONNET \& CHAPMAN 1956; KITCHING 1971; FISH \& CARPENTER 1982; WALKER et al. 1991). Em criadouros naturais, de acordo com AMERASINGHE \& MUNASINGHA (1988), a diversidade de espécies costuma ser mais elevada do que aquela encontrada em criadouros artificiais, tornando difícil uma análise de flutuação dessas populações, ao longo do tempo, levando em consideração todos esses fatores.

$\mathrm{O}$ acesso dos culicídeos nos entrenós só poderia ter ocorrido mediante furos que eram quadrados, retangulares ou circulares, podendo estes terem sido aumentados por ação de entrada e saída de outros insetos ou deteriorização da própria planta. LoUTON et al. (1996), em suas análises na planície Peruana, acreditavam que o responsável pelos furos, encontrados a 2,5 metros de altura, foi Leiobliastes laevis Beier, 1960 (Tettigoniidae). Os autores sugerem que a oviposição desse ortóptero ocorra durante o início do crescimento do bambu, quando a haste ainda é flexível. Davis (1944) coletando em bambu no Rio de Janeiro, encontrou furos feitos por besouro, com $0,25 \mathrm{~cm}$ de diâmetro. Constatou que existia preferência de algumas espécies, de acordo com a origem do furo. Furos circulares, quadrados e retangulares foram encontrados em taquaras Merostachys por LOZOVEI (1998), na Serra do Mar e no Primeiro Planalto do estado do Paraná, tendo sido constatado que os furos circulares foram elaborados por Eucalyptra barbara Schaus, 1894 e Eucalyptra fumida Schaus, 1894 (Noctuidae, Ophederinae).

Nesta pesquisa foram encontrados dois tipos de furos: circulares (1 a $4 \mathrm{~mm}$ de diâmetro) e retangulares $(3 \times 8 \mathrm{~mm}$ a $5 \times 10 \mathrm{~mm})$, além de furos grandes, abertos 
por ação de entrada e saída de outros insetos ou deteriorização como pode ser observado na (Fig. 5). As taquaras apresentaram diâmetro interno médio de 2,00; 1,$90 ; 1,64 ; 1,36 ; 1,12 \mathrm{~cm}$ respectivamente em $1,2,3,4$ e 5 metros de altura, onde era realizado o corte, com o auxílio de facão.

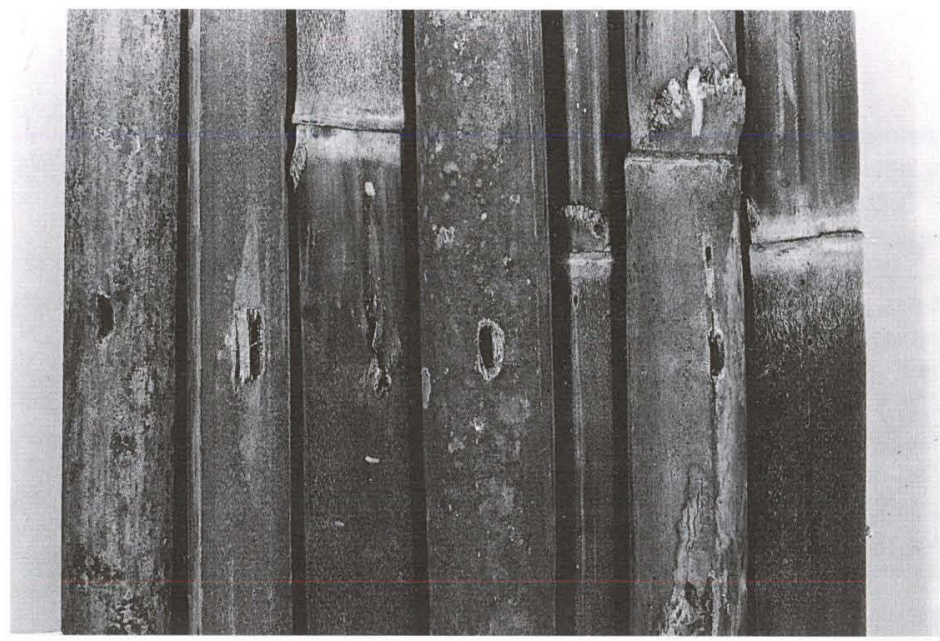

Fig. 5. Diversos padrões de furos encontrados em taquara Merostachys sp. na mata Daher, localizada na área urbana de Londrina, Paraná.

No interior desses entrenós, foram encontrados larvas e adultos de Desmosomus longipes Perty, 1830 (Curculionidae, Molytinae). Este besouro adulto, encontrado livremente na natureza, foi freqüente no interior dos entrenós e como apresenta peças bucais mastigadoras instaladas no ápice de uma projeção da cabeça, o que leva a sugerir ser responsável por um dos padrões de furo aqui obsevados. Este inseto foi também observado por CosTA LiMA (1956) em entrenós de bambu, e, além de D. longipes, observou também Dionychus paralleogramus Germar, 1829; Astyage lineigera Pascoae, 1873, Neoerethistes lateralis (Boh., 1836) (Erethistes lateralis (Boh., 1836)) com hábitos de brocar o lenho.

\section{CONCLUSÕES}

As reservas de matas em áreas urbanas e periurbanas, protegidas por lei, já fazem parte do contexto urbanístico por proporcionarem o bem-estar da população. Este tipo de ecossistema é repleto de diferentes habitats para inúmeros animais que mereciam um estudo pormenorizado. É importante conhecer a proliferação das espécies de mosquitos nestas reservas. A presente investigação foi conduzida somente em um dos habitats formados por entrenós de taquara. Nestes microhabitats, encontrou-se dez espécies de culicídeos. Nenhuma das espécies encontradas apresentou densidade populacional suficiente para oferecer risco de veicular agentes patogênicos ao homem. 
AGRADECIMENTOS. À CAPES pelo auxílio financeiro, através da bolsa de mestrado. A equipe do Prof. Dr. Oswaldo Paulo Forattini, Faculdade de Higiene e Saúde Pública de São Paulo, pela confirmação das espécies identificadas. Ao Prof. Dr. Sérgio Vanin (USP), pela identificação do Curculionidae. À Profa. Dra. Inês de Batista Fonseca, Universidade Estadual de Londrina (UEL), pela orientação nos testes estatísticos. À Profa. Dra. Ana Leuch Lozovei, (UFPR), e ao Prof. Claúdio Muller (UEL), pela revisão do artigo.

\section{REFERÊNCIAS BIBLIOGRÁFICAS}

Amerasinghe, F.P. \& N.B. Munasingha. 1988. A predevelopment mosquito survey in the Mahacueli development project área, Sri Lanka, immatures. Jour. Med. Entomol. 25 (4): 286-294.

Bonnet, D.D. \& H. ChAPMAN. 1956. The importance of mosquito breeding in tree holes with special reference to the problem in Tahiti. Mosquito News.16 (4): 301-305.

Borror, J.D. \& DeLong. 1969. Estudo dos insetos. São Paulo, EDUSP, 653p.

Costa Lima, A. 1956. Coleopteros, quarta e última parte, Insetos do Brasil. Rio de Janeiro, Escola Nacional de Agronomia, Vol. 10,373p.

DAvIS, D.E. 1944. Larval habitats of some brazilian mosquitoes. Rev. Entomol. 15 (1-2): 221-235.

FiSH, D. \& S.R. CARPENTER. 1982. Leaf litter and larval mosquito dynamics in tree-hole ecosystems. Ecology 63 (2): 283-288.

Forattini, O.P. 1965. Entomologia Médica. São Paulo, EDUSP, Vol. 3, 416p.

Jenkins, D.W. \& S.J. CARPENTER. 1946. Ecology of the tree hole breeding mosquitoes of Neartic North America. Ecol. Monogr. 16: 33-47.

Kitching, R.L. 1971. An ecological study of water-filled tree-holes and their position in the woodland ecosystem. Jour. Anim. Ecol. 40: 281-302.

KNight, K.L. \& A. Stone. 1977. A catalog of mosquitoes of the world (Diptera: Culicidae). Baltimore, Entomol. Soc. Amer., 611 p.

LANE, J. 1953. Neotropical Culicidae. São Paulo, EDUSP, Vols 1-2, 1111 p.

LOPES, J. 1997. Ecologia de mosquitos (Diptera: Culicidae) em criadouros naturais e artificiais de área rural do norte do estado do Paraná, Brasil. V. Coleta de larvas em recipientes artificiais instalados em mata ciliar. Rev. Saúde Pública 31 (4): 370 -377.

ـ 1999. Ecologia de mosquitos (Diptera, Culicidae) em criadouros naturais e artificiais de área rural do norte do Paraná, Brasil. VIII. Influência das larvas predadoras (Toxorhynchites sp.; Limatus durhamii e Culex bigoti) sobre a população de larvas de Culex quinquefasciatus e Culex eduardoi. Revta bras. Zool. 16 (3): 821-826.

Louton, J.; J. Gelhaus \& R. Bouchard. 1996. The aquatic macrofauna of water-filled bamboo (Poaceae: Bambusoideae: Guadua) internodes in a Peruvian lowland tropical forest. Biotropica 28 (2): $228-242$.

Lozovel, A.L. 1998. Mosquitos dendrícolas (Diptera, Culicidae) em internódios de taquara da floresta atlântica, serra do mar e do primeiro planalto, Paraná, Brasil. Brazil. Archs. Biol. Technol., Curitiba, 41: 501-508.

Lozover, A. L. \& E. Luz. 1976. Diptera Culicidae em Curitiba e arredores. I. Ocorrência. Arq. Biol. Tecnol., Curitiba, 19: 25-42.

MacDonald, W.W. \& R. TRAuB; 1960. Malaysian parasites XXXVIII. An introduction to the ecology of the mosquitoes of the lowland dipterocarp forest of Selangor, Malaya. Stud. Inst. Med. Res. Malaya. 29: 79-110.

Machado-Allison, C.E. 1980. Ecologia de los mosquitos (Culicidae) I. Huevos y oviposicion. Acta Biol. Venez. 10 (3): 303-371.

SotA, T. \& M. Mogi. 1996. Species Richness and altitudinal variation in the aquatic metazoan community in bamboo phytotelmata from north Sulawesi. Res. Popul. Ecol. 38 (2): 275-281.

WaLKer, E.D.; D.L. LANSON; R.W. MERRITT; W.T. Morgans \& M.J. KLUG. 1991. Nutriente dynamics, bacterial populations, and mosquito productivity in tree hole ecosystems and microcosms. Ecology 72 (5): 1529-1546.

Recebido em 01.V1.2000; aceito em 18.V.2000.

Revta bras. Zool. 18 (2): 429 - 438, 2001 